\title{
Éditorial : Négligence envers les aînés au travail : organisations de la société civile, âgisme et retraite obligatoire
}

\section{Introduction}

Dans le film de Lucino Visconti Mort à Venise (1971), un compositeur plus très jeune est troublé par un bel adolescent. La première scène du film offre un contraste puissant entre l'infortuné imprésario et le débardeur bien musclé de plus de soixante-dix ans qui charge les lourds bagages du touriste angoissé et le met en garde contre un gondolier sans scrupule. La séquence magique de Visconti capture l'essence d'une personne du troisième âge très fière d'être encore au travail en ce début de l'ère industrielle, à une époque où une grande majorité de citoyens de plus de 65 ans travaillaient encore, et aussi pendant la période où se déroule le scénario, avant l'insidieux mélange de l'idéologie de la retraite et de l'âgisme qui a évacué la plupart des employés plus âgés des lieux de travail.

Je désire insister sur la réponse insuffisante des organisations de la société civile (OSC) ${ }^{1}$ à l'étonnante disparition, depuis un siècle, de la voix des aînés $\mathrm{du}$ milieu du travail. Permettez-moi de vous en donner un exemple frappant. À la Nouvelle-Orléans, la rupture des digues après le passage de l'ouragan Katrina a eu des effets dévastateurs sur les aînés, qui ont souffert de façon disproportionnée : noyade, malnutrition, bouleversement des personnes déplacées, maladie et perte de biens. Malgré tout, le site Web de la sixième International Conference on Diversity in Organisations, Communities and Nations (2006) - aussi touché par l'ouragan (il a dû se transporter de la Xavier University envahie par les inondations à l'hôtel Sheraton New Orleans) - omet de mentionner l'âge dans sa définition de différence («ethnicité, sexe, race, socioéconomique, autochtone, religion, genre [sic], orientation sexuelle, incapacité» sont les catégories soulignées) et, à la conférence même, ni présentations ni groupes de discussion n'ont été consacrés aux aînés. Si l'on traverse la moitié du continent, le Congrès de la Fédération canadienne des sciences humaines (2006) a occulté l'âge de sa section sur "l'équité», et la liste nominale de dizaines de présentations portant sur les questions féminines contourne le sujet, bien qu'une grande partie des aînés soient des femmes.
Ce ne sont pas des anomalies, mais des cas courants de la règle indiquant que les préoccupations en matière d'équité, en matière d'emploi et la diversité au sein de la société civile sont peu orientées vers ceux qui ont dépassé l'âge de 60 ou 65 ans. Les États-Unis sont les chefs de file en ce qui a trait à l'âgisme, principalement grâce à l'American Association of Retired Persons (AARP) et la U.S. Equal Economic Opportunity Commission - qui administre la Loi sur la discrimination fondée sur l'âge en matière d'emploi (Age Discrimination in Employment Act - ADEA). Mais seulement un quart de la population américaine active de plus de 65 ans est au travail (en effet, l'ADEA ne s'applique pas aux bureaux de direction, où l'on s'attend à ce que les cadres prennent leur retraite à 65 ans ou avant). Les organismes gouvernementaux qui soutiennent ces travailleurs plus âgés (comme les commissions des droits de la personne) et les organismes de défense des droits des aînés dans d'autres pays développés ont peu de pouvoir ou n'existent tout simplement pas. (La plupart des autorités administratives qui s'occupent des "aînés" se concentrent sur des moyens visant à aider les personnes plus tout à fait jeunes et donc, de ce fait, vulnérables à compenser pour leur fragilité ou leur perte d'autonomie.)

\section{La loi et la retraite obligatoire}

Il n'existe aucune loi exigeant la retraite obligatoire. Cette pratique repose sur l'absence de protection juridique des travailleurs âgés face aux employeurs et aux syndicats. Les plus ardents praticiens de la retraite forcée comprennent notamment les universités, censées être les défenseurs de l'équité et de la justice sociale. Lorsque les États-unis ont aboli la retraite obligatoire en 1986, le secteur postsecondaire a livré une campagne vigoureuse pour en être exempté. La loi américaine sur la discrimination fondée sur l'âge en matière d'emploi (Age Discrimination in Employment Act) est finalement entrée en vigueur en 1994 (cette initiative est encore en vigueur) et les collèges américains ont lancé des programmes de retraite anticipée et des régimes bonifiés afin d'ouvrir grande la porte aux 
professeurs âgés. Si la carotte ne fonctionne pas, les administrateurs brandissent le bâton. Donc, l'University of Maryland at College Park a aboli cette année le populaire et tout à fait unique cours (sur la mort et le deuil) dispensé aux aînés par Daniel Leviton, 75 ans, et lui a ordonné d'enseigner aux étudiants de première année - une manoeuvre de découragement pratiquée dans d'autres collèges (voir «Hell, No - He Won't Go», 2006).

La loi très attendue de l'Union européenne promettant d'interdire la retraite obligatoire au plus tard en 2006 a présenté des clauses nationales d'exception facilement négociables par un camion de 5 tonnes. Confronté à l'opposition rebelle de la puissante Confederation of British Industry, qui a monté une campagne de publicité «work till you drop» («travailler à coeur joie»), le gouvernement Blair a mis de côté en décembre dernier ses plans d'application des droits inhérents au milieu de travail après 65 ans. $^{2}$ Bien que les organismes de défense des personnes du troisième âge comme Age Concern aient vainement protesté, la plupart des groupes britanniques de société civile sont demeurés cois.

An Canada, sauf au Manitoba et au Québec (les deux provinces ont aboli la retraite forcée au début des années 80), les travailleurs font face à une série de politiques en matière de retraite obligatoire. Appuyé par le Syndicat canadien de la fonction publique, par exemple, la Bibliothèque municipale de Saskatoon a renvoyé la bibliothécaire à temps partiel Louise Carlson en 2003 en raison de son âge. Son cas fait maintenant péniblement son chemin dans les dédales du Tribunal des droits de la personne de la Saskatchewan (Saskatchewan Human Rights Tribunal, 2005). Le couperet s'abattra bientôt sur Ian Whishaw, titulaire de la chaire de recherche en neurosciences $\mathrm{du}$ Bureau des gouverneurs de l'Université de Lethbridge, parce qu'il a célébré trop d'anniversaires (University of Alberta et al. 2005.). Tout en promettant de tenir compte des recommandations de sa Commission des droits de la personne (qui a fait volte-face sur la question de la retraite obligatoire en 2004) (voir Commission des droits de la personne du Nouveau-Brunswick, 2005), le gouvernement du Nouveau-Brunswick n'a pas encore présenté de loi visant à éliminer cette pratique.

Le gouvernement de l'Ontario a lancé à l'automne 2004 un processus de consultation visant à éliminer la retraite obligatoire. Au tout début de l'année, l'Université de Toronto a aboli la retraite forcée, en raison d'une grave pénurie de candidats à l'emploi et de la perte des professeurs plus âgés, afin de faire concurrence aux institutions anglo-américaines (la Nouvelle-Zélande et l'Australie ont cessé d'imposer la retraite obligatoire au cours des années 90). L'entente de Toronto a probablement incité Queen's Park à ignorer les demandes pressantes du secteur universitaire pour une dérogation à la loi visant à éliminer la retraite forcée (mais le gouvernement de l'Ontario a accordé un an de grâce avant la proclamation de la loi le 12 décembre 2006).

Les initiatives de l'Ontario ranimeront probablement la lutte du Canada contre la discrimination fondée sur l'âge, après un hiatus de 15 ans imposés aux cas devant la Cour suprême et visant les bibliothécaires, les médecins et les professeurs d'université. Dans le cas de la désastreuse décision McKinney en 1990, les savants juges ont rejeté la demande - entre autres de Bernard Blishen, directeur de la recherche de la Commission Hall qui avait établi le régime d'assurance-maladie du Canada et était le principal fondateur de la sociologie au Canada. Un homme de loi de 67 ans a débouté la chercheure métis Olive Dickason lors du jugement Dickason en 1992. À 50 ans, Madame Dickason s'était jointe à l'Université de $l^{\prime}$ Alberta et avait sans aucune aide établi la discipline de l'histoire autochtone. Maintenant âgée de 86 ans, Madame Dickason est encore troublée par cette décision. Elle a récemment fait remarquer «que la politique [de la retraite obligatoire] a davantage tendance à jouer contre les femmes que contre les hommes, puisqu'elles élèvent leurs familles avant de devenir titulaires d'un doctorat» ( $U$ of A Rethinks Policy on Mandatory Retirement, 2006).

\section{Les OSC canadiennes et la retraite obligatoire}

Le classique de Robert N. Butler Why Survive? (1975), réagissait fermement contre les mauvais traitements réservés aux aînés américains dans leur milieu de travail, et l'élégant Age Discrimination and the Mandatory Retirement Controversy (1988) de Martin Lyon Levine, a mis en contexte ce problème pour les Américains. Mais il faut revenir à The Final Plateau (1974) de Daniel Jay Baum pour découvrir une puissante sortie canadienne contre la discrimination fondée sur l'âge en milieu de travail. ${ }^{3}$ La question de l'âgisme - même le mot en soi - est presque absente du mouvement syndical au Canada. Une étude fédérale a révélé que «la plupart des syndicats appuient - ou au moins acceptent - la retraite obligatoire comme moyen d'offrir des possibilités d'emploi (c'est-à-dire des promotions) et la sécurité d'emploi (c'est-à-dire moins de mises à pied) à leurs jeunes membres. Cette situation est particulièrement vraie durant les périodes de repli économique» (Fourzly et Gervais, 2004). 
Suivant un modèle similaire d'autres pays développés, la plupart des OSC au Canada, y compris les organismes des droits de la personne et des libertés civiles, offrent peu d'opposition au principe de la retraite obligatoire. Lorsqu'un professeur de 74 ans a lutté contre sa retraite forcée de l'Université de Winnipeg l'an dernier (certaines universités du Manitoba pratiquent maintenant la retraite obligatoire grâce à une exemption pour les institutions postsecondaires accordée au milieu des années 90), un «spécialiste des droits de la personne» a mené la bataille contre lui (Macafee, 2006). La recherche effectuée par le Centre canadien de politiques alternatives (CCPA) (2006), un groupe de réflexion progressiste, parle peu de l'âgisme. ${ }^{4}$

Comme leurs contreparties américaines, les organisations féministes canadiennes n'ont pas de position claire en ce qui a trait à l'âgisme. (La U.S. National Women Studies Association a analysé le problème de l'âgisme pour la première fois lors de son Congrès historique en 2005. Ailleurs dans le paysage du féminisme américain, les aînées sont presque invisibles [voir Marshall, 2006].) Women's Legal Education and Action Fund (LEAF) (n.d.[a]), qui surveille les effets de la Charte canadienne des droits et libertés sur l'égalité des femmes, ne mentionne aucune poursuite en matière de retraite obligatoire parmi ses cas importants (bien que sur son site Web il y ait une section spéciale pour les «Adolescents» [voir LEAF n.d.(b)]). "Présentations du LEAF au Comité de révision de la Loi canadienne sur les droits de la personne, Novembre 1999» n'a pas abordé la retraite forcée, bien que le document ait indiqué que les femmes se voient souvent assigner «le rôle de dispensatrices non rémunérées de soins aux enfants, aux hommes et aux personnes âgées» (FAEJ, 1999, p. 24).

Un manque de sensibilisation à l'âgisme chez les OSC féministes et autres OSC a des conséquences désastreuses, non seulement dans le cadre de ces organisations, mais aussi dans l'ensemble des collectivités plus larges sur lesquelles elles exercent leur influence. Sans cette reconnaissance de la part du milieu universitaire, par exemple, on peut seulement jusqu'à un certain point s'interroger sur l'âgisme et sur la détermination des conditions requises pour libérer les aînés des contraintes difficiles à supporter (Liscomb, 2006).

\section{Perspectives en matière d'égalité des aînés en milieu de travail}

L'institution moderne de la retraite peut être au coeur de notre idéologie contemporaine de l'âgisme comme une détérioration et un déclin inévitable.
Il faut remarquer que la grande expérience de la retraite a généré peu de recherche sur «ce que la vieillesse devrait signifier» (Achenbaum, p. 50). Néanmoins, un thème dominant de la philosophie occidentale est la valeur du travail pour l'esprit humain. Forcer la capacité d'une personne à rejeter le monde extérieur pour satisfaire des besoins sociaux contribue à la santé et à la croissance individuelles, à n'importe quel âge. Bonnie Rooks est à 78 ans la métallurgiste la plus âgée des États-unis et elle travaille dans un milieu physiquement exigeant non seulement parce qu'elle a besoin d'argent pour sa fille handicapée mais aussi pour ce que le travail signifie pour elle. Elle s'inquiète de ce qu'elle fera si elle démissionne. "J'ai de la chance, j'ai des tas de jeunes amis. Mais ils seront tous au travail et je ne sais pas ce que je pourrai bien faire de moi-même» (At 78, Bonnie Rooks Likes a 'Dirty Old Job' in an Ohio Steel Mill, 2005).

À l'encontre de la plupart des organisations de la société civile, le gouvernement et les organismes responsables de la politique industrielle en sont venus à apprécier la nature des pertes sociétales importantes qui pourraient être encourues si on laisse aller à la dérive les travailleurs plus âgés (voir, par exemple, Organisation de coopération et de développement économiques, 2005): non seulement le besoin de soutien financier d'une multitude de retraités qui pourront vivre encore 20 ou 30 ans mais aussi les pénuries possibles sur le marché du travail associées au gaspillage éhonté des compétences et du savoir des employés plus âgés dans une population active qui vieillit rapidement.

Un nouveau programme en milieu de travail encouragerait la flexibilité au travail et une plus grande autonomie pour tous les travailleurs, jeunes et vieux. Il permettrait de prolonger la vie active en combattant les stéréotypes discriminatoires qui subsisteront certainement après l'abolition de la retraite obligatoire. Les employeurs fourniraient des avantages en matière d'avancement et de formation, peu importe l'âge. Pour aider à concrétiser cette stratégie de changement, les organisations de la société civile doivent faire face à leurs attitudes discriminatoires et repenser la nature du travail.

J'ai commencé cet essai par un portrait cinématique d'une personne dans la force de l'âge, et il serait approprié de conclure par une autre illustration tirée d'un long métrage plus récent. Parlant du rôle qu'il a joué dans le film controversé primé par un Oscar Million Dollar Baby (2004), Clint Eastwood a fait remarquer que le gérant de boxe de plus de 70 ans Frankie Dunn «était retraité dans sa tête» avant la rencontre fatidique du boxeur de 32 ans plein de 
promesse des Ozarks, Maggie Fitzgerald (Eastwood, 2005). Le film est une parabole du savoir perdu et retrouvé, du mentorat intergénérationnel, et de l'inspiration mutuelle et de l'appui obtenu lors d'un conflit de travail. Il peu faire partie d'une compréhension interculturelle transformée, et attendue depuis longtemps, de l'âge et du travail à l'aube du deuxième millénaire.

\section{David MacGregor}

Professeur de sociologie

Collège universitaire King's à l' Université de Western Ontario

\section{Notes}

1 Aux fins du présent essai, voici une définition utile des organisations de la société civile (OSC):

Les acteurs de la société civile comprennent notamment les forums d'affaires, les associations religieuses, les mouvements syndicaux, les groupes communautaires locaux, les organisations non gouvernementales (ONG), les fondations philanthropiques, les groupes de réflexion ... [Les partis politiques [ne sont pas inclus] dans la société civile, étant donné que contrairement aux autres groupes qui viennent d'être cités - les partis politiques visent à occuper des fonctions publiques. Les médias ne sont pas non plus pris en considération. (Fonds monétaire international, 2003).

Bien que l'attention qu'ils portent à ce sujet soit au mieux de nature sporadique, les médias (par ex., journaux, télévision, radio) voient d'un oeil plus sympathique les demandes des travailleurs plus âgés que la plupart des OSC.

2 La législation du gouvernement sur la retraite obligatoire est actuellement en appel devant cinq Lords juristes dans un cas portant sur deux travailleurs masculins plus âgés. (voir Test Case on Redundancy Rights for Over 65s Goes to the Lords, 2006).

3 Mes collègues C.T. Gillin et Thomas Klassen (2000) ont rompu une longue période de silence par une série d'articles, notamment Retire Mandatory Retirement (voir aussi Gillin, MacGregor et Klassen, 2005).

4 Toutefois, le Monitor du Centre canadien de politiques alternatives (CCPA) a publié un éditorial de cet auteur sur la retraite obligatoire (accompagné d'une réfutation) dans son numéro de Décembre 2005-Janvier 2006 (voir MacGregor, 2005/2006).

\section{Références}

Achenbaum, W.A. What is retirement for? (2006). Wilson Quarterly, 30(2), disponible au http://www. wilsoncenter.org/index.cfm?fuseaction $=$ wq.essay\& essay_id=178668, consulté le 27 juin 2006.
At 78, Bonnie Rooks likes a "dirty old job" in an Ohio steel mill. (10 août 2005). Wall Street Journal, p. A1.

Butler, R.N. (1975). Why survive: Being old in America. Baltimore: John Hopkins University Press.

Centre canadien de politiques alternatives. (2006). CCPA reports and studies, disponible au http:// www.policyalternatives.ca/index.cfm?act $=$ main\&call $=$ A2286B2A\&pa $=$ A2286B2A\&type $=5 \&$ office_ID $=2,3,1,4$, $5,6 \& d o=$ list\&sr $=151$, consulté le 6 juin 2006.

Fédération canadienne des sciences humaines. (2006). Congress 2006, disponible au http://www.fedcan.ca/ congress2006/, consulté le 27 juin 2006.

Dickason c. University of Alberta [1992] 2 S.C.R. 1103 (S.C.C.).

Eastwood, C. (producteur et directeur) et P. Haggis (producteur). (2004). Million Dollar Baby [long métrage]. États-Unis: Warner Bros.

Fourzly, M. et Gervais, M. (2004). Chapitre 5 : (Anti-) discrimination clauses and practices. Collective agreements and older workers in Canada, disponible au site Web de Développement social Canada: http:/ / www.sdc.gc.ca/en/lp/spila/wlb/caowc/ 10chapter_5.shtml\#bnote4-, consulté le 31 mai 2006.

Gillin, C.T. et Klassen, T. (juillet-août 2000). Retire mandatory retirement. Policy options, disponible au http:// www.irpp.org/po/archive/jul00/gillin.pdf, consulté le 27 juin 2006.

Gillin, C.T., MacGregor, D. et Klassen, T. (2005). Time's up: Mandatory retirement in Canada, Toronto: James Lorimer.

Hell, no-He won't go. (4 mai 2006). Inside Higher Education, disponible au http://insidehighered.com/layout/set/ print/news/2006/05/04/maryland, consulté tiré le 27 juin 2006.

Fonds monétaire international. (2003). Définition: Qu'est-ce que la société civile? Guide for staff relations with civil society organizations, disponible au http://www. imf.org/external/np/cso/eng/2003/101003.htm\#II, consulté le 6 juin 2006 .

Levine, M.L. Age discrimination and the mandatory retirement controversy. Baltimore: John Hopkins University Press, 1988.

Macafee, M. (2005). University of Winnipeg prof must take mandatory retirement for now. Canadian Press Newswire, disponible au http://www.recorder.ca/cp/National/ 050817/n081799A.html.

MacGregor, D. (2005/2006). Yes, right to work is fundamental, even for people over 65. CCPA Monitor: Economic, Social, and Environmental Perspectives, 12(7), 24.

Marshall, L. (2006). Aging: A feminist issue. NWSA Journal, 18(1), vii-xiii.

McKinney c. University of Guelph, [1990] 3 S.C.R. 220 (S.C.C.). 
Commission des droits de la personne du NouveauBrunswick. (2005). Guideline on mandatory retirement, disponible au http://www.gnb.ca/hrc-cdp/e/ Guideline-on-Mandatory-Retirement.pdf, consulté le 27 juin 2006.

Lipton, J. (2005). Takes on Three [entrevue avec Clint Eastwood]. Dans C. Eastwood (directeur et producteur) et P. Haggis (producteur), Million Dollar Baby (2 disques DVD, éd. grand écran) [long métrage spécial]. États-Unis: Warner Bros.

Liscomb, V.B. (2006). We need a theoretical base : Cynthia Rich, women's studies and ageism [entrevue avec Cynthia Rich]. NWSA Journal, 18(1), 3-12.

Organisation de coopération et de développement économiques. (2005). Vieillissement et politiques de l'emploi: Canada. Paris: Auteur.

Tribunal des droits de la personne de la Saskatchewan. (14 octobre 2005). Décision concernant la demande d'un intervenant, disponible au http://www.saskhrt.ca/ forms/index/Decisions/101405.htm, consulté le 27 juin 2006.

Sixième International Conference on Diversity in Organizations, Communities and Nations. (2006). Thème 2006: Human rights, diversity and social justice, disponible au http:/ /d06.cgpublisher.com/themes.html, consulté le 6 juin 2006.
Test case on redundancy rights for over $65 \mathrm{~s}$ goes to the Lords. (6 mars 2006). Guardian, disponible au http:// www.guardian.co.uk/law/story/0,,1724385,00.html, consulté le 6 juin 2006.

$\mathrm{U}$ of A rethinks policy on mandatory retirement. (23 mai 2006). Edmonton Journal, disponible au http://www. canada.com/edmontonjournal/news/story.html?id= 7c55060f-4959-4072-a598-1f94f90c28b2\&k=87997\&p=2, consulté le 6 juin 2006.

Visconti, L. (producteur et directeur). (1971). Mort à Venise [long métrage]. États-Unis: Warner Bros.

University of Alberta et al. (2005). Ian Whishaw. Biography, disponible au http://www.psych.ualberta.ca/GCPWS/ kolb_whishaw.html, consulté le 27 juin 2006.

Women's Legal and Education and Action Fund. (1999). Présentation au Comité de révision sur la Loi canadienne sur les droits de la personne, novembre 1999, disponible au http://www.leaf.ca/legal-pdfs/ CHRAReviewSubmission99.PDF, consulté le 27 juin 2006.

Women's Legal and Education and Action Fund. (n.d.[a]). Cas importants. Legal work, disponible au http:// www.leaf.ca/legal-cases.html, consulté le 27 juin 2006.

Women's Legal and Education and Action Fund. (n.d.[b]). No means no: Equality rights, responsibilities and the Charter. Teen pages, disponible au http:/ / www.leaf.ca/ teen-no.html consulté le 27 juin 2006. 


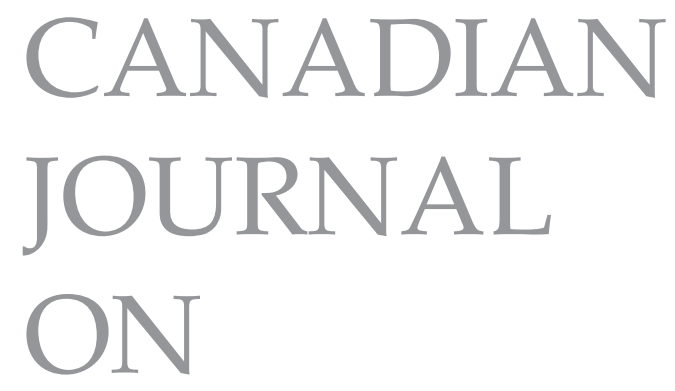

AGING

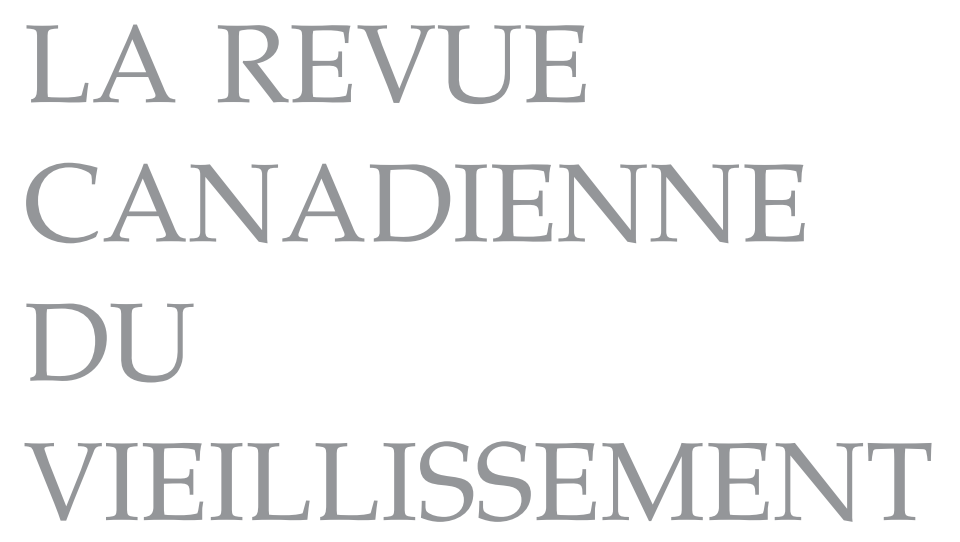

